\title{
A Novel Noncontact Ultrasonic Levitating Bearing Excited by Piezoelectric Ceramics
}

\author{
He Li, Qiquan Quan *, Zongquan Deng, Yuxiang Hua, Yinchao Wang and Deen Bai \\ State Key Laboratory of Robotics and System, Harbin Institute of Technology, Harbin 150001, China; \\ lihe_hit@163.com (H.L.); dengzq@hit.edu.cn (Z.D.); huayuxiang_hit@163.com (Y.H.); \\ wangyinchao2008@126.com (Y.W.); baideen@163.com (D.B.) \\ * Correspondence: quanqiquan@hit.edu.cn; Tel.: +86-451-8641-3857
}

Academic Editor: Sheng-Yuan Chu

Received: 1 September 2016; Accepted: 23 September 2016; Published: 1 October 2016

\begin{abstract}
A novel ultrasonic levitating bearing excited by three piezoelectric transducers is presented in this work. The transducers are circumferentially equispaced in a housing, with their center lines going through the rotation center of a spindle. This noncontact bearing has the ability to self-align and carry radical and axial loads simultaneously. A finite element model of the bearing is built in ANSYS, and modal analysis and harmonious response analysis are conducted to investigate its characteristics and driving parameters. Based on nonlinear acoustic theory and a thermodynamic theory of ideal gas, the radical and lateral load-carrying models are built to predict the bearing's carrying capacity. In order to validate the bearing's levitation force, a test system is established and levitating experiments are conducted. The experimental data match well with the theoretical results. The experiments reveal that the maximum radical and axial levitating loads of the proposed bearing are about $15 \mathrm{~N}$ and $6 \mathrm{~N}$, respectively, when the piezoelectric transducers operate at a working frequency of $16.11 \mathrm{kHz}$ and a voltage of $150 \mathrm{~V}_{\mathrm{p}-\mathrm{p}}$.
\end{abstract}

Keywords: noncontact bearing; near-field levitation; squeeze film; piezoelectric transducer

\section{Introduction}

Many sophisticated power machines and equipment such as high-speed precision machine tools, aero-engines, and micro gas turbines (MGT) demand greater bearing performance in terms of high-speed capacity, precision, reliability, longevity, low noise, etc. [1-3]. Traditional contact bearings hardly can meet these requirements due to problems such as wear, heat generation and so on [4]. Therefore, noncontact bearings have been extensively studied. The main types of noncontact bearings widely adopted today include hydrostatic bearings, hydrodynamic bearings, and electromagnetic-suspension bearings. However, these bearings also have limitations [5]. Gas pumps and pipelines are absolutely necessary for hydrostatic bearings, which results in high operation costs and bulky systems [6]. Serious wear will appear in the starting or stopping stage of hydrodynamic bearings, and an eccentricity phenomenon is inevitable [7]. With regard to electromagnetic-suspension bearings, demagnetization and magnetic leakage will affect their performance. Besides, a complicated control system is needed to guarantee their high precision and stability [8]. Hence, improvement of existing bearing technology and development of novel bearing concepts are of great significance.

As a promising alternative solution, the ultrasonic levitating bearing based on near-field acoustic levitation (NFAL) was proposed $[9,10]$. This kind of bearing carries a load using acoustic radiation force excited by piezoelectric ceramics. Compared with other noncontact bearings, air-supply devices and lubrication are not needed. In the starting or low-speed stage, ultrasonic bearing can generate identical carrying capacity, ensuring good stability. Bearing clearance and carrying capacity can be adjusted through changing the amplitude of the vibration surface. The control system of an ultrasonic 
bearing is simple, and does not cause electromagnetic interference to sensitive electronic components in the system. The levitation height of ultrasonic bearings can be controlled at the micron level, which can meet precision requirements. Moreover, the friction is very low as the bearing operates at a high speed, which makes it feasible to raise the speed limit [11-13].

Benefiting from the development of piezoelectric ceramics technology, a lot of ultrasonic bearing concepts and structures excited by piezoelectric ceramics have come into existence. Yoshimoto et al. first proposed a novel ultrasonic linear bearing based on elastic hinges and piezoelectric ceramic stacks. Although elastic hinges can magnify the vibration amplitude under excitation of PZT (piezoelectric ceramics of $\mathrm{PbZr03-PbTiO3} \mathrm{system)} \mathrm{stacks,} \mathrm{they} \mathrm{also} \mathrm{bring} \mathrm{about} \mathrm{structural} \mathrm{complexity} \mathrm{and} \mathrm{high}$ machining costs $[14,15]$. Based on Yoshimoto's concepts, Stolarski improved the design. The improved linear bearing can suspend an object weighing $800 \mathrm{~g}$ at a height of a few micrometers $[16,17]$. Stolarski and Ha et al. presented a new kind of ultrasonic journal bearing, which integrates ultrasonic levitation with hydrodynamic lubrication aimed at solving the wear problem of aerodynamic bearings in the starting or low-speed stage. The force required from one piezo-actuator to produce deformation of lobe depth of $3.5 \mu \mathrm{m}$ was about $10 \mathrm{~N}$ [18-20]. Chao Wang proposed a tubular squeeze-film journal bearing. The bearing was designed to be driven at its 13th mode at the frequency of $16.37 \mathrm{kHz}$ at which the amplitude response was $3.22 \mu \mathrm{m}$ [21-23]. Su Zhao presented an active journal air bearing actuated by high-power piezoelectric transducers. A steel spindle with a diameter of $50 \mathrm{~mm}$ had been successfully levitated and driven at low rotational speed. Each of the three ultrasonic transducers can provide a load carrying force up to $51 \mathrm{~N}$ at an input power of $50 \mathrm{~W}$ [24,25]. Zhigang Yang et al. focused the study on supporting patterns of ultrasonic bearings and successfully developed ultrasonic thrust bearings, bidirectional supported ultrasonic bearings and hybrid-levitation bearings integrating ultrasonic levitation into gas bearings [26-28]. Chao Chen proposed a noncontact spherical bearing to explore a potential supporting method for gyroscopes. A maximum revolution speed of about $1071 \mathrm{r} / \mathrm{min}$ was measured for the prototype $[29,30]$.

A novel noncontact ultrasonic levitating bearing that can simultaneously carry radical and lateral loads is first put forward in this paper. The bearing is excited by a radiator with a slot structure that can convert a sandwich transducer's unidirectional vibration into bidirectional vibration. Furthermore, this bearing has the function of self-aligning, which helps to improve the stability of the bearing. The finite element analysis method is adopted to obtain the bearing's main performance parameters. To predict the bearing's carrying capacity, radical and lateral radiation force models are established. In the radical radiation force model, the non-uniformity of air film and the surface roughness effect are first taken into consideration. Also, the thermodynamic theory of ideal gas is used to estimate the lateral radiation force. Eventually, experiments are conducted to validate the presented bearing's capacity to support radical and lateral loads.

\section{Structure and Operating Principle}

The proposed bearing consists of three piezoelectric transducers (PT-1, PT-2, and PT-3) mounted on a housing. As shown in Figure 1a, the transducers are set circumferentially, and their center lines' intersection angle is 120 degree between each other. The center lines of all the transducers go through the rotation center of the spindle. Each transducer proposed has a radiator in the front of the horn. The radiator has a concave surface which covers about 110 degrees of the spindle surface. A " $\|$ | "-shaped slot through the lateral section is designed in order to transform partial vertical longitudinal vibration into flexural vibration on the radiator's two lateral surfaces when the transducer is driven to vibrate at its first longitudinal resonance frequency. As a result of the near-field acoustic levitation effect, squeeze films emerge between radiator's concave or lateral surface and the spindle's surface. A repelling force in the perpendicular direction is generated to suspend the spindle and automatically keeps it at an equilibrium position. Similarly, a thrust force in a lateral direction is generated to bear the axial load. Since the levitation force increases when the bearing clearance is decreased, the spindle will be dragged back to the center as it deviates from the bearing bore's center. This active self-aligning technology 
characteristic can guarantee the ultrasonic bearing will operate at a very steady state. The working principle of the proposed bearing is shown in Figure 1b.

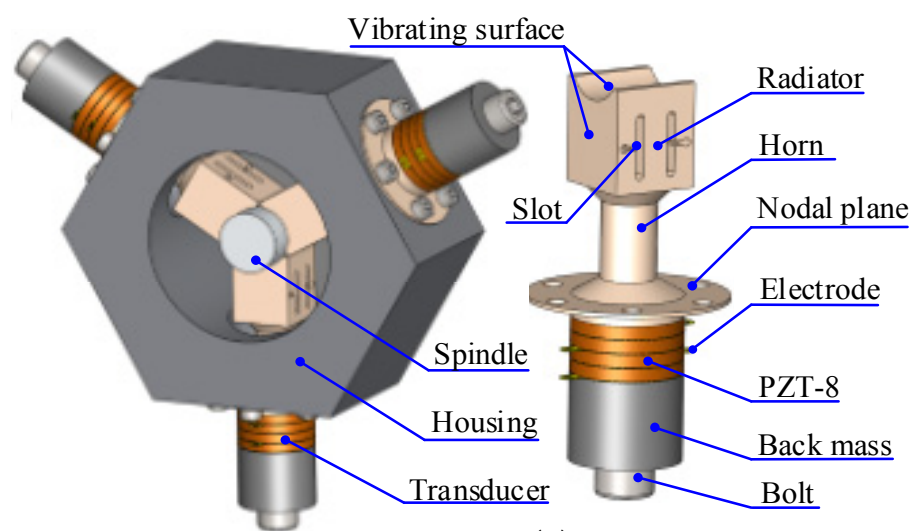

(a)

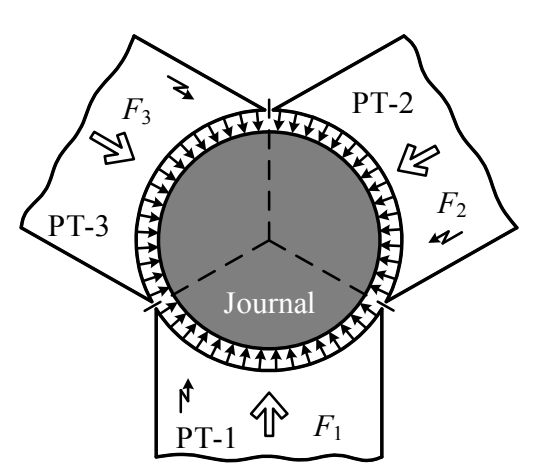

(b)

Figure 1. Structure of the proposed noncontact bearing: (a) three-dimensional model of the ultrasonic bearing and transducer's constitution; (b) working principle of ultrasonic levitating bearing.

\section{Working Frequency and Output Vibrating Amplitude of the Transducer}

Using a commercially available FEM package (ANSYS12.0, ANSYS Inc., Canonsburg, PA, USA), the resonance frequency of the transducer is determined by modal analysis. The material of the horn and radiator is titanium alloy with a mass density of $4430 \mathrm{~kg} / \mathrm{m}^{3}$, Young modulus of $1.15 \times 10^{11} \mathrm{~N} / \mathrm{m}^{2}$ and Poisson ratio of 0.32 . The material of the back mass is stainless steel with mass density of $7930 \mathrm{~kg} / \mathrm{m}^{3}$, Young modulus of $2.09 \times 10^{11} \mathrm{~N} / \mathrm{m}^{2}$ and Poisson ratio of 0.3 . The material of the ceramic is PZT-8. The relative dielectric permittivity matrix $[\varepsilon]$ at constant strain, piezoelectric stress matrix $[e]$ and elastic coefficient matrix $[c]$ are:

$$
\begin{gathered}
{[\varepsilon]=\left[\begin{array}{ccc}
919 & 0 & 0 \\
0 & 919 & 0 \\
0 & 0 & 826
\end{array}\right] \times 10^{-11} \mathrm{~F} / \mathrm{m} \quad[e]=\left[\begin{array}{ccc}
0 & 0 & -4.1 \\
0 & 0 & -4.1 \\
0 & 0 & 14.0 \\
0 & 0 & 0 \\
0 & 10.3 & 0 \\
10.3 & 0 & 0
\end{array}\right] \mathrm{C} / \mathrm{m}^{2}} \\
{[c]=\left[\begin{array}{cccccc}
14.9 & 8.11 & 8.11 & 0 & 0 & 0 \\
0 & 14.9 & 8.11 & 0 & 0 & 0 \\
0 & 0 & 13.2 & 0 & 0 & 0 \\
0 & 0 & 0 & 3.4 & 0 & 0 \\
0 & 0 & 0 & 0 & 3.13 & 0 \\
0 & 0 & 0 & 0 & 0 & 3.13
\end{array}\right] \times 10^{10} \mathrm{~N} / \mathrm{m}^{2}}
\end{gathered}
$$

The block Lanczos method recommended by ANSYS instructions is chosen to calculate transducers' modes. The first-order longitudinal mode is an available mode, as shown in Figure 2. The calculated resonant frequency of first-order longitudinal mode is $16.241 \mathrm{kHz}$.

In order to obtain the amplitude on vibrating surface of radiator, harmonic analysis is conducted in a frequency domain which covers the first-order longitudinal vibrating frequency. According to the modal analysis result, the frequency domain is set between $16 \mathrm{kHz}$ and $16.5 \mathrm{kHz}$. Given the energy loss when transducer is driven by an ideal voltage generator, the transducer's structural damping ratio $(0.3 \%)$ is taken into consideration. Figure 3 shows the exciting method and structural constraint in harmonic analysis. The input peak voltage is $150 \mathrm{~V}$ during finite element simulation. 


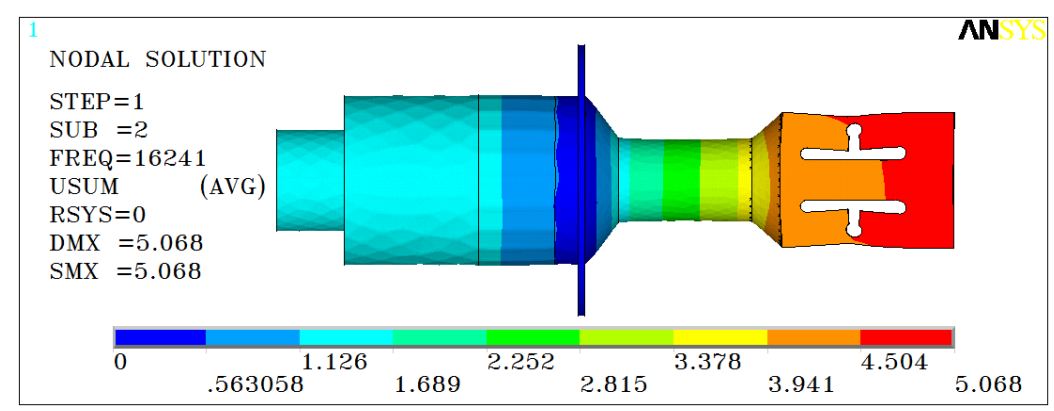

Figure 2. The first-order longitudinal mode of the transducer at $16.241 \mathrm{kHz}$.

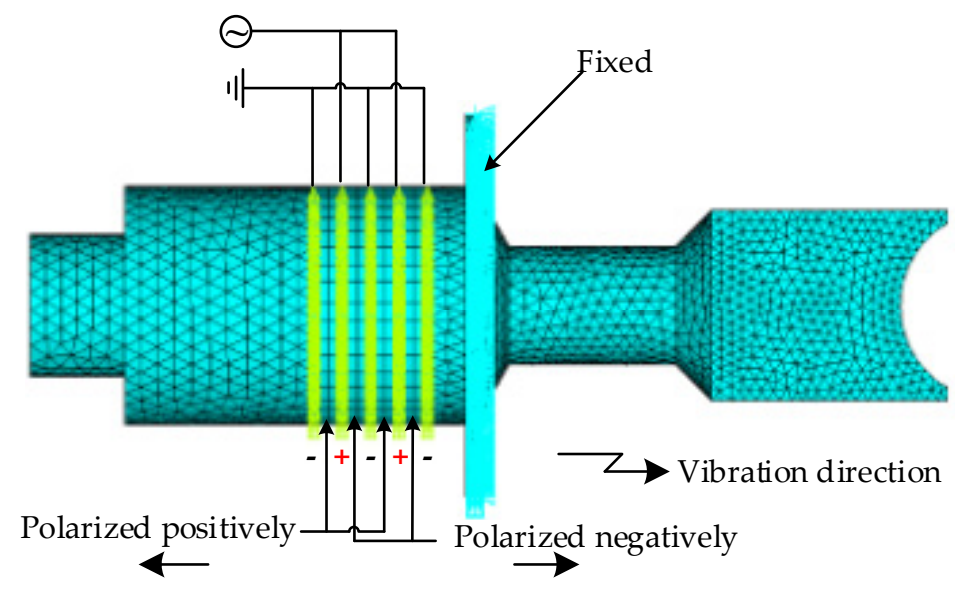

Figure 3. Exciting method and structural constraint for harmonic analysis.

The results of harmonic analysis are shown in Figure 4. The nodes numbered 45693 and 5537, which are near the center of the concave surface and in the thinnest area of the lateral surface, respectively, are chosen to be calculated. It is clearly seen that the maximum amplitude of Node 1 is approximately $12 \mu \mathrm{m}$ at the resonant frequency, while that of Node 2 is about $3.5 \mu \mathrm{m}$.

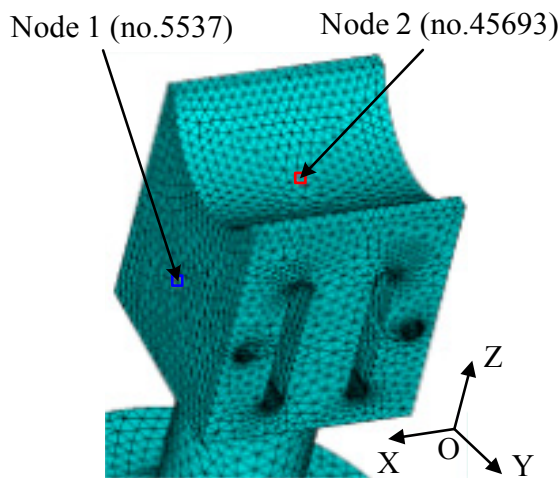

(a)

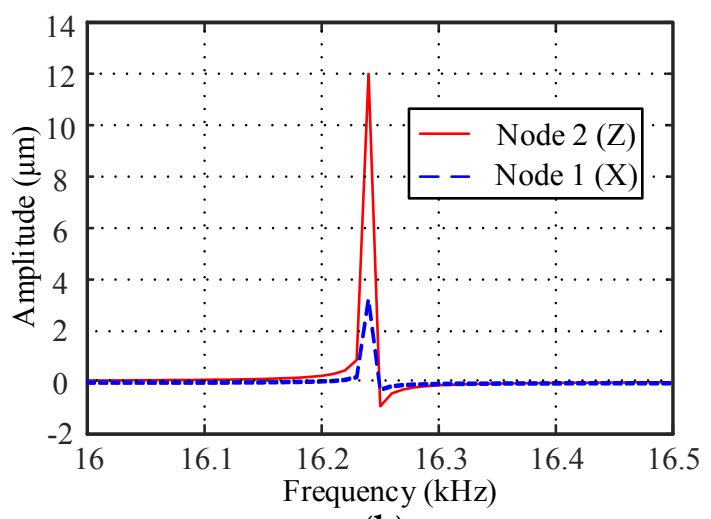

(b)

Figure 4. Nodes chosen to be calculated and their simulation results: (a) nodes on concave surface and lateral surface; (b) vibrating amplitude of Node 1 in $x$-direction and Vibrating amplitude of Node 2 in z-direction. 


\section{Load-Bearing Capacity of a Single Transducer}

Owing to the near-field acoustic levitating effect, the high-frequency vibration on the radiator's concave surface and lateral surface will produce an acoustic radiation force on the supported objects. By means of calculating a single transducer's radiation forces in the radical and lateral directions, the load-carrying capacity of the ultrasonic bearing can be determined. In this section, the radical and transverse mechanical models are established using the nonlinear acoustic theory and thermodynamics theory of ideal gas, respectively.

\subsection{Modeling of Radical Radiation Force}

Figure 5 shows the cross section of a radical levitation structure including a journal and a radiator excited by single peizoelctric transducer. The concave surface with a radius $R$ and width $L$ vibrates at a resonance frequency $f$ and a journal with a radius $r$ is placed above at a height $h_{0}$. The layer of air between the concave surface and journal surface is conventionally called gas squeeze film. The order of the air squeeze film thickness is $10^{-5} \mathrm{~m}$, which is three orders smaller than the dimensions of the vibrating surface $R$ and $L$ (with order of $10^{-2} \mathrm{~m}$ ). Thus, an acoustic wave generated by vibrating surface can be considered an approximate plane wave, and the air film pressure gradient along the thickness direction could be ignored. Since the suspended journal is a rigid body, the deformation will not occur, and the journal will exert no impact on the sound field. In return, air squeeze film pressure cannot change the modes of the vibrating surface.

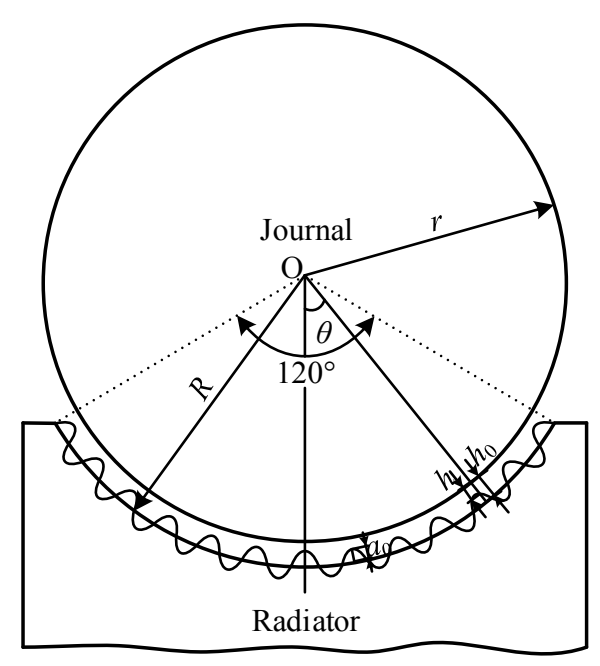

Figure 5. Cross-section diagram of radical levitation structure driven by a single transducer.

The wave equation of a one-dimensional sound wave can be written as

$$
\frac{\partial^{2} p}{\partial t^{2}}=c^{2} \nabla^{2} p
$$

where $p$ represents the acoustic pressure, $t$ the time, $c$ acoustic velocity, and $\nabla$ Laplace operator. Considering the multiple reflection between transducer's radiation surface and the journal's cylindrical surface, the sound pressure $p$ and velocity $v$ of waves in $z$-direction are calculated as

$$
\left\{\begin{array}{l}
p=2 A \cos k z \cos \omega t \\
v=\frac{2 A}{\rho_{0} c} \sin k z \sin \omega t
\end{array}\right.
$$

where $A$ is the amplitude of acoustic pressure before stacking of incident sound waves and reflected sound waves, $k$ the wave number, $\rho_{0}$ the density of air, and $\omega$ the angular frequency. Since the heat 
propagation velocity in the air is much lower than the acoustic velocity, the ultrasonic levitating process can be considered adiabatic. In addition, there is little acoustic energy dissipation caused by thermal conduction and viscous force among air molecules in the air squeeze film, so acoustic absorption in levitating process is also neglected. Based on the theory of thermodynamics, kinetic energy density $\bar{K}$, potential energy density $\bar{V}$ and total acoustic energy density $\bar{E}$ after time-averaging in the bearing's air squeeze film are derived as

$$
\left\{\begin{array}{l}
\bar{K}=\frac{1}{2} \rho_{0}\left(\frac{1}{T} \int_{0}^{T} v d t\right)^{2}=A^{2} \sin ^{2} k z /\left(\rho_{0} c^{2}\right) \\
\bar{V}=\left(\frac{1}{T} \int_{0}^{T} p d t\right)^{2} \cos ^{2} k z /\left(2 \rho_{0} c^{2}\right)=A^{2} \cos ^{2} k z /\left(\rho_{0} c^{2}\right) \\
\bar{E}=\bar{K}+\bar{V}=A^{2} / \rho_{0} c_{0}^{2}
\end{array}\right.
$$

According to a study by Lee C. P. and Wang T. G. [31], the mean radiation pressure in an ideal gas is described as

$$
p_{\text {ra }}=\left(p-p_{0}\right)_{\text {avg }}=\bar{K}-\bar{V}+\frac{\gamma-1}{2} \bar{E}=\left(\frac{\gamma-1}{2}+\cos 2 k z\right) \frac{v_{0}^{2} \rho_{0}}{4 \sin ^{2} k h}
$$

Given $\cos 2 k z \approx 1, \sin k h \approx k h, a_{0}=\frac{v_{0}}{\omega}, k=\frac{\omega}{c}$, Equation (4) can be simplified as

$$
p_{\mathrm{ra}}=\left(p-p_{0}\right)_{\text {avg }}=\frac{\gamma+1}{8} \frac{a_{0}^{2} \rho_{0} c^{2}}{h^{2}}
$$

During the bearing's levitation process, when the journal is suspended at different heights $h_{0}$ in $z$-direction, the thickness $h$ of air film varies apparently along the circumferential direction. As shown in Figure 6, if levitation height $h$ is equal to the radius difference $\delta$ between the journal's cylindrical surface and the bearing's radiation surface, the thickness $h$ of air squeeze-film is uniform. However, if that is not the case, the thickness $h$ of air squeeze-film will change over the angle $\theta$ between $z$-direction and the line from the journal center $\mathrm{O}$ to any given point on the radiation surface. The non-uniformity of air squeeze-film's thickness will exert a significant effect on the ultrasonic bearing's levitation capacity.

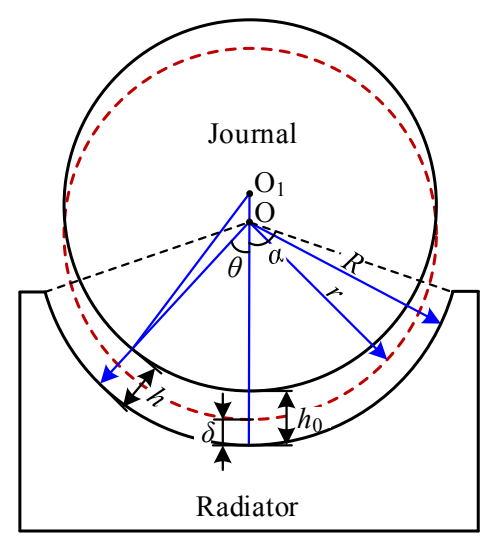

(a)

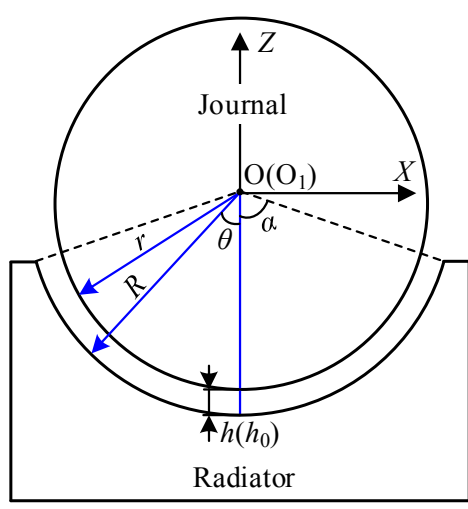

(b)

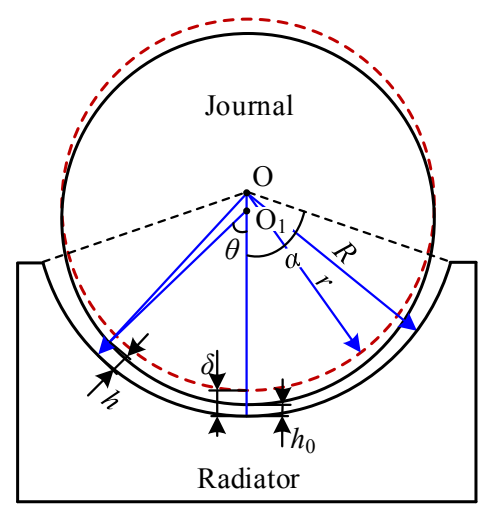

(c)

Figure 6. The distribution diagram of air squeeze-film's thickness at different heights: (a) $h_{0}>\delta$; (b) $h_{0}=\delta ;(\mathbf{c}) h_{0}<\delta$.

Through geometric analysis, the relationship between air film thickness $\mathrm{h}$ and position angle $\theta(\theta \in(-\alpha, \alpha))$ can be calculated. The thickness $h_{1}, h_{2}$, and $h_{3}$ respectively in case a, case $\mathrm{b}$, and case $\mathrm{c}$ are given by 


$$
\begin{cases}h_{1}(\theta)=R-\left(r \sin \left[\theta-\arcsin \left(\frac{h_{0}-\delta}{r} \cdot \sin \theta\right)\right] / \sin \theta\right) & h_{0}>\delta \\ h_{2}(\theta)=\delta & h_{0}=\delta \\ h_{3}(\theta)=R-\sqrt{\left(\delta-h_{0}\right)^{2}+r^{2}+2 r\left(\delta-h_{0}\right) \cos \theta} & h_{0}<\delta\end{cases}
$$

The surface roughness is ignored during the above-mentioned process of calculating air squeeze-film thickness. In the bearing-journal system, actually, the surface roughness of the bearing and journal is a key factor affecting the bearing's levitation capacity. If relative surface roughness, that is, the ratio between the surface roughness of the bearing or journal and the thickness of air film, is greater than $5 \%$, this effect should not be neglected. Surface roughness will exert a significant impact on levitating force, especially when air film thickness has the same order as surface roughness. Figure 7a shows the surface roughness of the bearing and journal. The surface roughness is assumed to have the form of long narrow ridges and valleys distributed in the circumferential direction and axial direction. Figure $7 \mathrm{~b}$ shows the surface topography of a region $90 \mu \mathrm{m} \times 90 \mu \mathrm{m}$ on the journal cylindrical surface, measured with an atomic force microscope. Figure $7 \mathrm{c}$ describes the relationship among air film thickness $h_{\mathrm{f}}$, surface topography parameter $\xi_{1}$ and $\xi_{2}$, and the gap $h$ between an ideal journal and radiation concave with smooth surface. Actually, when the journal contacts a radiation concave surface, the air film thickness $h_{\mathrm{f}}=0$, but the real gap $h=\xi_{1}+\xi_{2} \neq 0$. $\xi_{1}$ and $\xi_{2}$ represent the arithmetic mean value of all wave peaks on the journal and radiation concave surface, respectively. Since it is impractical to obtain all the wave peaks, several discrete regions on the journal and radiation surface are chosen to be measured, with the aim of obtaining $\xi_{1}$ and $\xi_{2}$. The expressions of $\xi_{1}$ and $\xi_{2}$ can be described as

$$
\left\{\begin{array}{l}
\xi_{1} \approx \frac{1}{m+n}\left(\sum_{i=1}^{m} Z_{1 \mathrm{v} i}+\sum_{j=1}^{n} Z_{1 \mathrm{v} j}\right) \\
\xi_{2} \approx \frac{1}{m+n}\left(\sum_{i=1}^{m} Z_{2 \mathrm{p} i}+\sum_{j=1}^{n} Z_{2 \mathrm{p} j}\right)
\end{array}\right.
$$

where $Z_{1 v i}$ and $Z_{1 v j}$ are peak values of all regions chosen on the journal surface in the axial direction and circumferential direction. respectively, $Z_{2 \mathrm{p} i}$ and $Z_{2 \mathrm{p} j}$ are peak values of regions chosen on the radiation concave surface in the axial direction and circumferential direction, respectively, and $m$ and $n$ are numbers of wave peaks in the axial direction and circumferential direction, respectively.

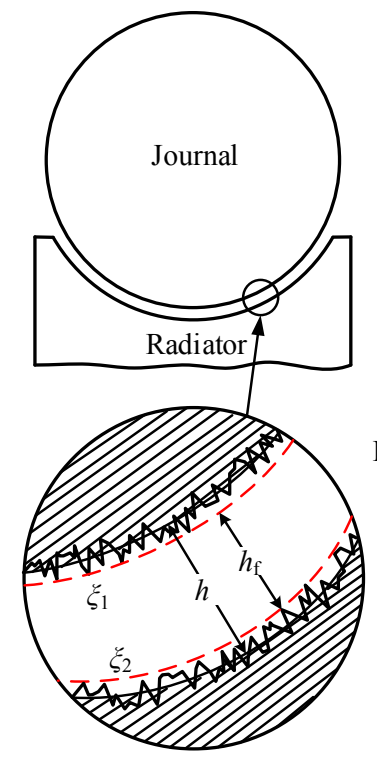

(a)

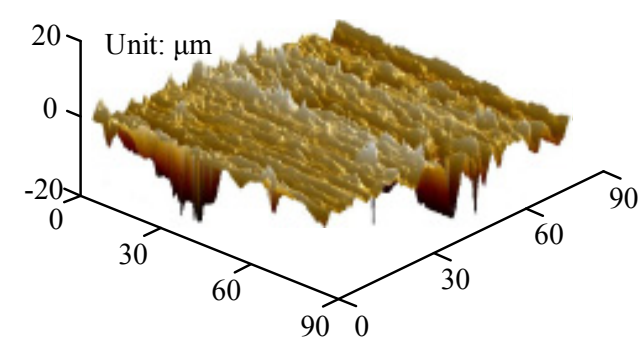

(b)

Ideal contour line Boundary line of air film

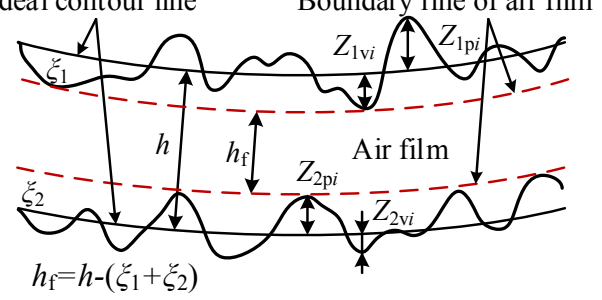

(c)

Figure 7. Surface roughness of bearing and journal: (a) physical construction of bearing; (b) surface topography of a region on the journal; (c) real film thickness $h$. 
Based on the above analysis, the radical levitation force of the ultrasonic bearing excited by each transducer can be calculated by Equation (8) which is as follows:

$$
F_{\mathrm{V}}=\iint_{S} \bar{p} \cos \theta \mathrm{d} S=\int_{-\alpha}^{\alpha} R \cos \theta \mathrm{d} \theta \int_{0}^{l} \bar{p} \mathrm{~d} x
$$

\subsection{Modeling of Lateral Radiation Force}

Different from radical levitation process above, the acoustic field excited by flexural vibration in lateral radiation process is hard to be described accurately. Both vertical and horizontal forces acting on an object that levitated on the vibrating surface were theoretically and experimentally studied by Hashimoto et al. [32-34]. In their studies, the levitation force was generated by longitudinal vibration which was considered to be piston motion, while the levitation force is excited by flexural vibration in our study. For this complicated case, the thermodynamic theory of ideal gas is adopted to approximately estimate the sound pressure in air film between the supported object and the radiation head's lateral surface.

Figure 8 shows different states of the lateral levitation process. The process includes two main stages: direct extrusion (see Figure 8b) and reverse extrusion (see Figure 8c). When the radiator's lateral surface vibrates at a high frequency, the air between the radiator and supported object will also be squeezed at a high frequency. Because of the viscosity of the gas, it is difficult for the air to flow in or out of the gap rapidly, so that the time-averaging pressure of air in the gap is higher than that of the ambient environment. Due to the existence of the pressure difference, an air squeeze film with a certain stiffness is generated.

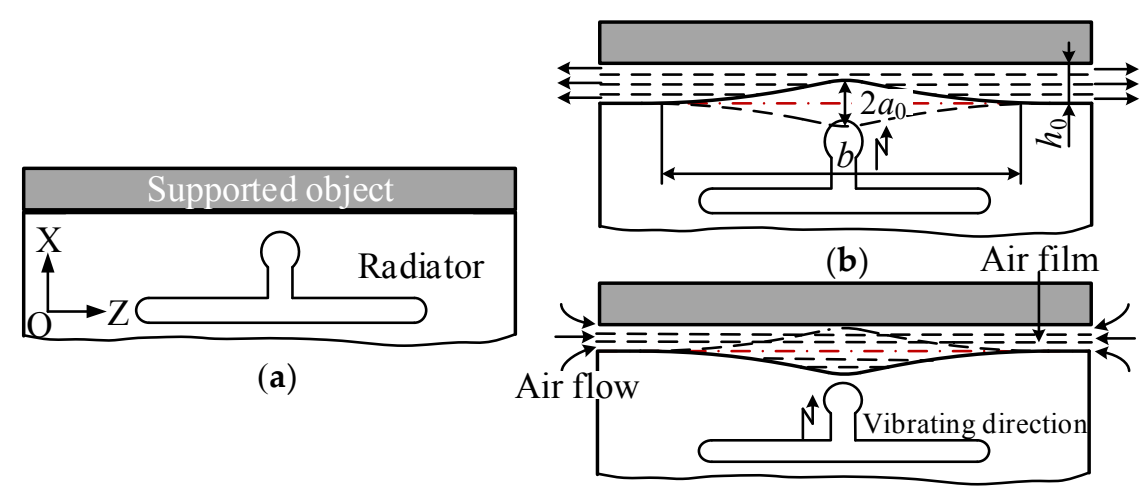

(c)

Figure 8. Lateral levitation process: (a) initial state; (b) direct extrusion; (c) reverse extrusion.

An assumption is made that the gas between two surfaces is perfectly ideal, and the heat that gas in the gap absorbs from or radiates to surroundings is neglected as the process of the air's condensation and expansion in the gap is very fast. Thus, according to the state equation of ideal gas in an adiabatic process, the relationship between ambient pressure $p_{0}$ and air film pressure $p$ can be expressed as

$$
\frac{p}{p_{0}}=\left(\frac{V_{0}}{V}\right)^{\gamma}
$$

where $V_{0}$ is the initial volume of air in the gap and $\gamma$ is a specific heat ratio $(\gamma=1.4$ for air).

The lateral surface of the radiator is subjected to the high-frequency excitation, and flexural vibration deformation occurs. The expression of flexural vibration is

$$
a=a_{0} \cos (\omega t)
$$


where $a_{0}$ and $a$ respectively represent the peak value and instantaneous value of amplitude and $\omega$ is angular frequency. If the thickness of air squeeze film is defined as $\bar{h}_{0}$, the overlapping area of radiator and levitated object is $S_{0}$, and the length and width of the flexural deformation area on the lateral surface are $l$ and $b$, then the approximate volume $V$ of air in the gap can be expressed as

$$
V \approx S_{0} \bar{h}_{0}-\frac{1}{2} a_{0} b l \cos (\omega t)
$$

So the mean pressure difference in a time period is derived as follows

$$
\bar{p}=\frac{1}{2 \pi} \int_{0}^{2 \pi} p \mathrm{~d}(\omega t)-p_{0}
$$

where $p=\frac{p_{0}}{\left(1-\frac{a_{0} b l \cos (\omega t)}{2 S_{0} \bar{h}_{0}}\right)^{\gamma}}$.

The lateral levitation force generated by the radiator is calculated by

$$
F_{\mathrm{L}}=\iint_{S} \bar{p} \mathrm{~d} S=\frac{1}{16} \frac{p_{0} \gamma(\gamma+1) a_{0}^{2} b^{2} l^{2}}{S_{0} \bar{h}_{0}^{2}}
$$

\section{Fabrication and Measurement}

Three prototypes of piezoelectric transducers are manufactured as shown in Figure 9a. An HP4294A impedance analyzer is used to obtain the resonance frequency and impedance characteristics. The maximum difference of minimum impedance frequency among the three transducers are less than $100 \mathrm{~Hz}$, which is within the error allowed. The mean values of the three transducers' test results are shown in Figure 9b. The error at the resonance frequency point between the test and the above simulation is mainly attributed to the machining tolerance, offset characteristics of PZTs, and the conductivity of the glue. In order to realizing impedance matching for ultrasonic power, an extra LC-type matching network needs to be cascaded in the circuit, which can adjust series resonance frequency to a certain point of the impedance curve with approximate zero phase angle.

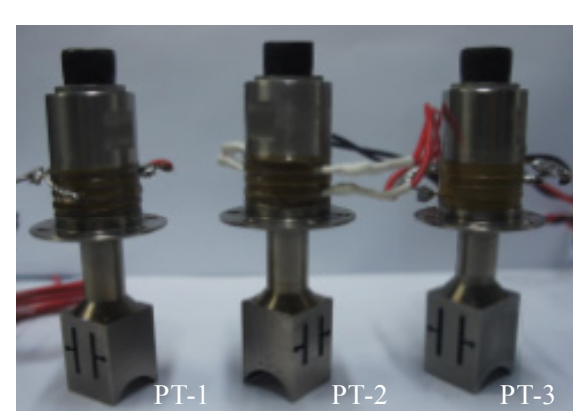

(a)

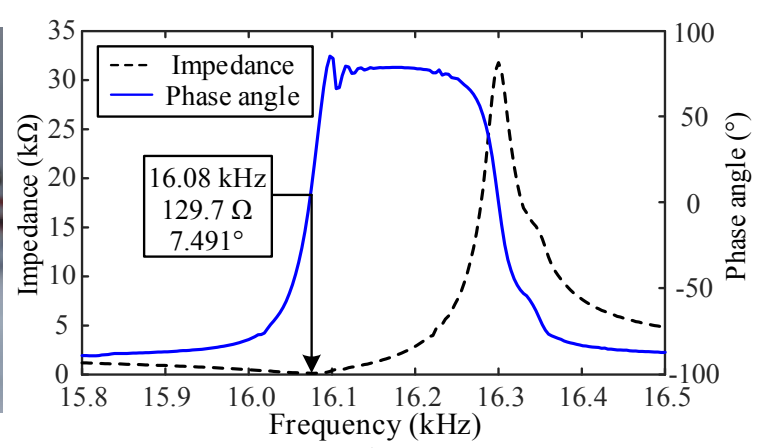

(b)

Figure 9. The piezoelectric transducer prototypes and the characteristic parameters: (a) transducer prototypes; (b) mean impedance and phase angle of the transducers.

A KEYENCE laser displacement sensor (LK-H020) is applied to obtain the vibration amplitude in the vertical and lateral directions. By changing the output frequency of the ultrasonic power supply, the resonance frequency can be easily determined. The measurement results shown in Figure 10 show that the maximum vertical and lateral vibration amplitudes are $10.8 \mu \mathrm{m}$ and $3.3 \mu \mathrm{m}$, respectively, where the frequency is about $16.11 \mathrm{kHz}$, which matches well with the simulation and the measurement 
results. The errors between the actual working frequency and results of simulation or the impedance analyzer measurement can be caused by many factors, but the different boundary conditions may exert the most significant influence.

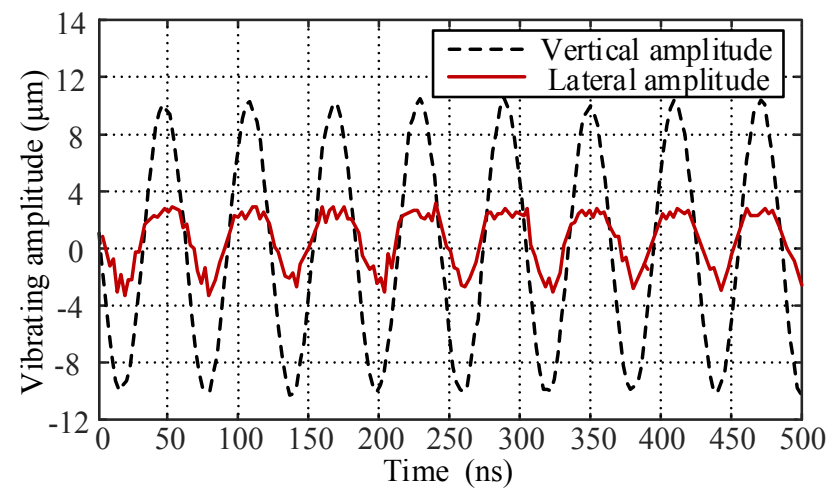

Figure 10. The vertical and lateral vibration amplitudes.

For the purpose of validating the levitation capacity of ultrasonic bearing excited by one piezoelectric transducer, a test system is established shown in Figure 11. The system mainly includes a vibration isolation platform, an ultrasonic bearing, weights, a supported object, a signal generator, a PZT driving amplifier, a laser displacement sensor and a computer, etc. The diameters of the ultrasonic bearing's concave surface and the journal's outer circle are $20.03 \mathrm{~mm}$ and $19.97 \mathrm{~mm}$ respectively. The mass of the journal is $23 \mathrm{~g}$. Different vertical and lateral loads can be acquired through changing the weights and supported objects with different masses. Sinusoidal voltage is set with an amplitude of $150 \mathrm{~V}_{\mathrm{p}-\mathrm{p}}$ in the test.

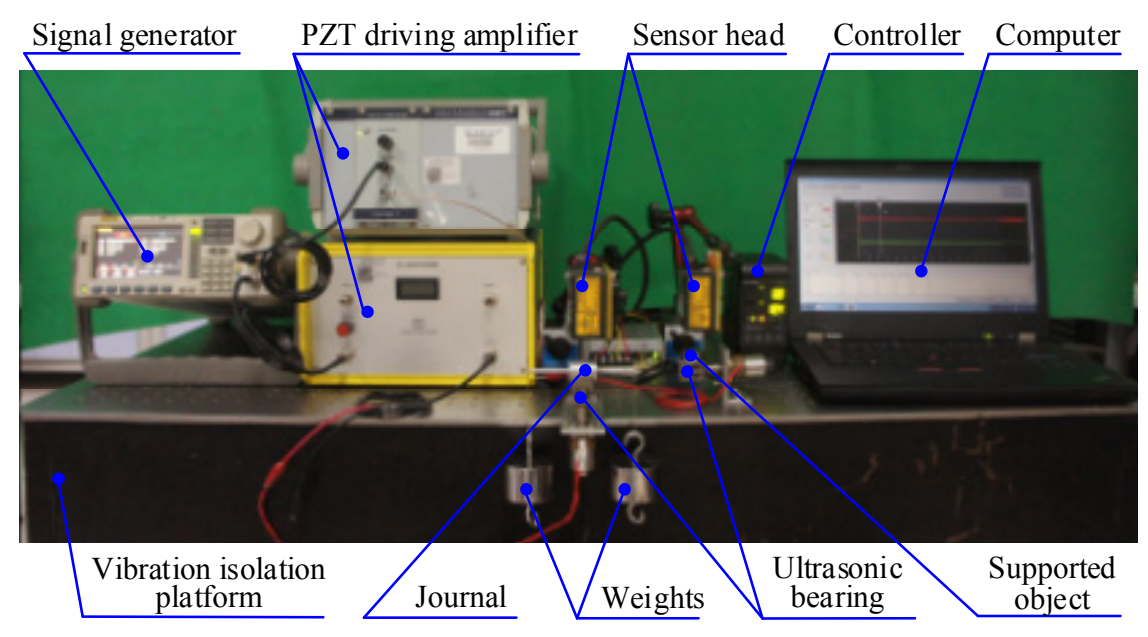

Figure 11. Load-carrying capacity test system.

The comparison between theoretical results and experiment data on radical levitation capacity is shown in Figure 12. It is clear that the levitation force is increased with the diminution of levitation height. When the levitation height is reduced to approximately the vibration amplitude of the radiation concave surface, the bearing force will reach the maximum value. In this case, the journal and radiation are in the critical contact state. The maximum radical levitation force is about $15 \mathrm{~N}$. The main factors limiting radical levitation capacity are the input power and surface machining quality. The input power directly influences the vibrating amplitude of the radiator's surface, and the load capacity will be improved significantly with the increase in vibrating amplitude. According to the radical levitation 
curve, surface roughness reveals a significant effect on levitation force, especially when levitation height is in a low value range.

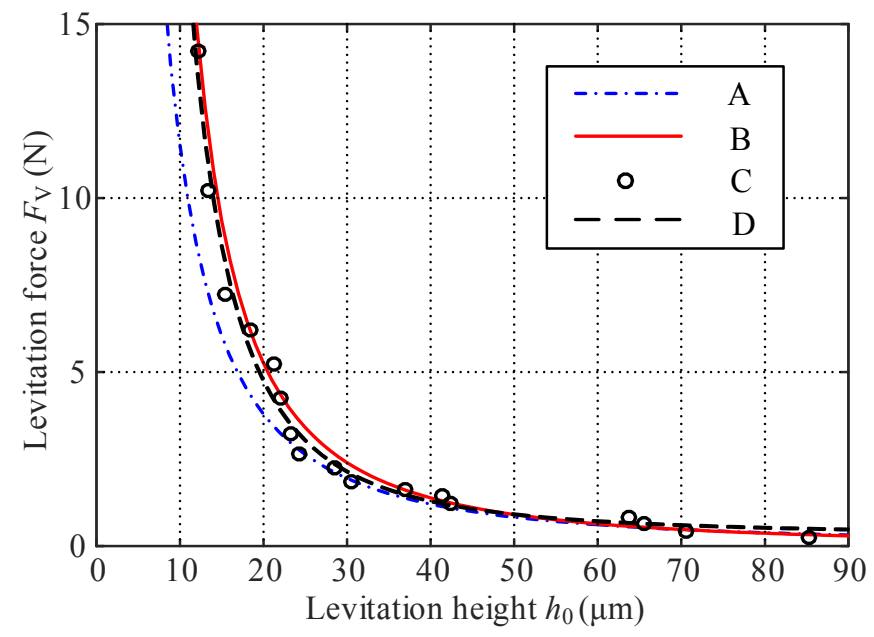

Figure 12. Radical levitation force predicted by different models and experimentally measured values. A-theoretical model with no surface roughness effect; B-theoretical model with surface roughness effect; C-experimental values; D—fitting curve of experimental data.

Here, the lateral levitation height under different bearing capacities was investigated as shown in Figure 13. The error between the theoretical model and experimental data is mainly ascribed to boundary effect, surface roughness, and the laser displacement sensor's measurement accuracy. Experimental data show that the maximum lateral levitation force is much smaller than the maximum vertical levitation force. This is because of the fact that the vertical levitation is excited by the longitudinal vibration of the whole concave surface while the lateral levitation is excited by flexural vibration of side faces, and the vertical vibration amplitude is higher than flexural vibration amplitude, which leads to the vibration energy in vertical direction is larger than that in lateral direction. The maximum lateral levitation force is about $2 \mathrm{~N}$. When the lateral force exceeds $2 \mathrm{~N}$, the bearing will contact with the supported object. Since the lateral vibration is at a high frequency, the bearing can generate friction-reducing effect even if the bearing and supported object come into contact, which has been demonstrated by many researchers $[35,36]$.

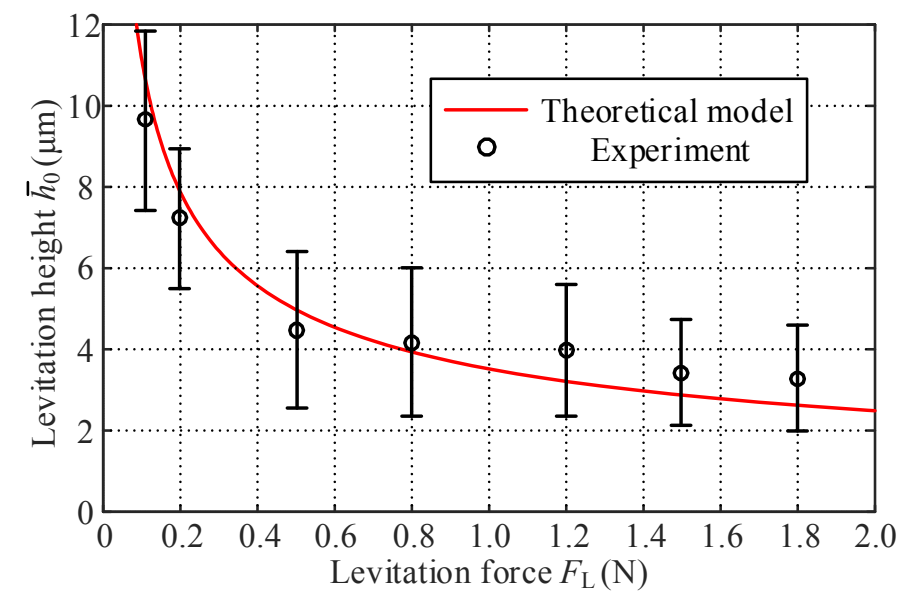

Figure 13. Comparison between theoretical lateral levitation force and experiment data. 


\section{Conclusions}

A novel concept for an ultrasonic levitating bearing actuated by piezoelectric transducers is proposed. The desired mode of vibration and amplitude of the vibrating surface are obtained through modal analysis and harmonic response analysis. The established theoretical models can be employed to estimate the bearing's levitation force. The experimental data show that the prototype transducer achieves a maximum radical load of $15 \mathrm{~N}$ and an axial load of $2 \mathrm{~N}$ under a working frequency of $16.11 \mathrm{kHz}$ and voltage of $150 \mathrm{~V}_{\mathrm{p}-\mathrm{p}}$. Thus, the bearing excited by three transducers can carry about $6 \mathrm{~N}$ in the axial direction. Furthermore, its load-carrying capacity in both the radial direction and axial direction can easily be increased by adding the input voltage of each transducer and improving the surface machining quality of the bearing and rotor. Compared with other ultrasonic bearings, the proposed ultrasonic bearing has a simpler fabrication than two-end bearings and supporting bearings; it also has better performance in terms of control, as the three transducers are virtually identical and can be controlled synchronously. Research on this kind of ultrasonic bearing can provide a new and better method for bidirectional supporting capacity.

Acknowledgments: This project is supported by the National Natural Science Foundation of China (No. 61403106); Program of Introducing Talents of Discipline to Universities (B07018); Fundamental Research Funds for the Central Universities (No.HIT.NSRIF.2014051).

Author Contributions: All authors conceived and designed the experiments and analyzed the data; He Li and Yuxiang Hua conducted the FEM analysis; Deen Bai and Yinchao Wang performed the experiments; all authors contributed to the writing of the paper; Qiquan Quan contributed to revising the paper.

Conflicts of Interest: The authors declare no conflict of interest.

\section{References}

1. Jiang, S.F.; Wang, X.L.; Yuan, Y.T. Characteristics and application technology of precision machine tool bearings. Bearing 2011, 7, 57-63.

2. Wang, Z.L.; Ge, Q.J.; Lin, G.C. Investigation on lubricating and cooling technologies for aeroengine mainshaft bearings. Aeroengine 2012, 38, 15-17.

3. Hikichi, K.; Togo, S.; Esashi, M.; Tanaka, S. Hydroinertia gas bearing for ultra-small gas turbine engine: Influence of iperating temperature for characteristic of hydroinertia gas bearing. J. Jpn. Soc. Tribol. 2010, 55, 292-299.

4. Mosleh, M.; Bradshaw, K. Role of component configuration in evaluation of accelerated rolling contact fatigue of ball bearings. Wear 2011, 271, 2681-2686. [CrossRef]

5. Khonsari, M.M.; Booser, E.R. Applied Tribology: Bearing Design and Lubrication; John Wiley \& Sons Ltd.: New York, NY, USA, 2008; pp. 1-21.

6. Wang, X.; Yamaguchi, A. Characteristics of hydrostatic bearing/seal parts for water hydraulic pumps and motors. Part 1: Experiment and theory. Tribol. Int. 2002, 35, 425-433. [CrossRef]

7. Asada, T.; Saitou, H.; Itou, D. Design of hydrodynamic bearing for mobile hard disk drives. IEEE Trans. Magn. 2005, 41, 741-743. [CrossRef]

8. Liu, S.J.; Fan, Y.; Di, J.; Liu, Y.J.; Qin, W.; Li, S. Nonlinear control approach of an electromagnetic bearing system. Appl. Mech. Mater. 2014, 538, 387-393. [CrossRef]

9. Ide, T.; Friend, J.; Nakamura, K.; Ueha, S. A non-contact linear bearing and actuator via ultrasonic levitation. Sens. Actuators A 2007, 135, 740-747. [CrossRef]

10. Nakamura, K. Noncontact transport of planner object through near field acoustic levitation. J. Acoust. Soc. Jpn. 2013, 69, 603-608.

11. Liang, Y.D.; Ling, H.; Zhang, Y. Study on the conditions of near-field acoustic levitation. Adv. Mater. Res. 2010, 97-101, 4135-4140. [CrossRef]

12. Ma, X.; Wang, T.; Wang, S. Research on the transient behavior of near field ultrasonic levitation during lifting up. Acta Acust. 2014, 39, 93-98.

13. Jia, B.; Chen, C.; Zhao, C. Solution on near-field acoustic levitation force in flexural mode based on ALE methods. Piezoelectr. Acoustoopt. 2013, 35, 80-84. 
14. Kazato, A.; Yoshimoto, S. Study on aerodynamic sliding table using squeeze-gas film effect. Trans. Jpn. Soc. Mech. Eng. 2000, 66, 3462-3468. [CrossRef]

15. Yoshimoto, S.; Kobayashi, H.; Miyatake, M. Floating characteristics of a squeeze-film bearing for a linear motion guide using ultrasonic vibration. J. Tribol. Int. 2007, 40, 503-511. [CrossRef]

16. Stolarski, T.A.; Woolliscroft, S.P. Performance of a self-lifting linear air contact. Proc. Inst. Mech. Eng. C J. Mech. Eng. Sci. 2007, 221, 1103-1115. [CrossRef]

17. Stolarski, T.A. Numerical modeling and experimental verification of compressible squeeze film pressure. Tribol. Int. 2010, 43, 356-360. [CrossRef]

18. Ha, D.N.; Stolarski, T.A.; Yoshimoto, S. An aerodynamic bearing with adjustable geometry and self-lifting capacity. Part 1:self-lift capacity by squeeze film. Proc. Inst. Mech. Eng. Part J: J. Mech. Eng J. Eng. Tribol. 2005, 219, 33-39. [CrossRef]

19. Stolarski, T.A. Acoustic levitation-A novel alternative to traditional lubrication of contacting surfaces. Tribol. Online 2014, 9, 164-174. [CrossRef]

20. Stolarski, T.A. Running characteristics of aerodynamic bearing with self-lifting capability at low rotational speed. Adv. Tribol. 2011, 2011. [CrossRef]

21. Wang, C.; Au, Y.H.J. Study of design parameters for squeeze film air journal bearing-Excitation frequency and amplitude. Mech. Sci. 2011, 2, 147-155. [CrossRef]

22. Wang, C.; Au, Y.H.J. Levitation characteristics of a squeeze-film air journal bearing at its normal modes. Int. J. Adv. Des. Manuf. Technol. 2012, 60, 1-10. [CrossRef]

23. Wang, C.; Au, Y.H.J. Comparative performance of squeeze film air journal bearings made of aluminum and copper. Int. J. Adv. Des. Manuf. Technol. 2013, 65, 57-66. [CrossRef]

24. Zhao, S.; Twiefel, J.; Wallaschek, J. Design and experimental investigations of high power piezoelectric transducers for a novel squeeze film journal bearing. In Proceedings of the Active and Passive Smart Structures and Integrated Systems, San Diego, CA, USA, 8 March 2009; Volume 7288, pp. 1-8.

25. Zhao, S.; Mojrzisch, S.; Wallaschek, J. An ultrasonic levitation journal bearing able to control spindle center position. Mech. Syst. Signal Process. 2013, 36, 168-181. [CrossRef]

26. Chang, Y.; Wu, B.D.; Yang, Z.G. Bearing capacity and anti-friction behavior of ultrasonic vibration bearing. J. Jilin Univ. Technol. 2004, 34, 222-225.

27. Tian, F.J.; Che, X.H.; Yang, Z.G.; Jiang, B.; Yao, X.F. Structure design of bidirectional support ultrasonic levitation bearing. Opt. Precis. Eng. 2009, 4, 813-818.

28. Liu, J.J.; Jiang, H.; You, H.; Jiao, X.Y.; Liu, J.F.; Yang, Z.G. Bearing capacity of ultrasonic levitation and pneumatic suspension. J. Xi'an Jiaotong Univ. 2013, 47, 56-60.

29. Chen, C.; Wang, J.S.; Jia, B.; Li, F. Design of a noncontact spherical bearing based on near-field acoustic levitation. J. Intell. Mater. Syst. Struct. 2014, 25, 755-767. [CrossRef]

30. Chao, C.; Wang, J.S.; Tong, L. Investigation on structural dynamic design and suspending characteristics of ultrasonic levitation type of gyros. J. Vib. Meas. Diagn. 2013, 33, 1060-1064.

31. Matsuo, E.; Koike, Y.; Nakamura, K.; Ueha, S.; Hashimoto, Y. Holding characteristics of planar objects suspended by near-field acoustic levitation. Ultrasonics 2000, 38, 60-63. [CrossRef]

32. Ueha, S.; Hashimoto, Y.; Koike, Y. Non-contact transportation using near-field acoustic levitation. Ultrasonics 2000, 38, 26-32. [CrossRef]

33. Yoshimoto, S.; Sekine, H.; Miyatake, M.; Yoshimoto, S.; Sekine, H.; Miyatake, M. A non-contact chuck using ultrasonic vibration: Analysis of the primary cause of the holding force acting on a floating object. Proc. Inst. Mech. Eng. C J. Mech. Eng. 2010, 224, 305-313. [CrossRef]

34. Chu, B.T.; Apfel, R.E. Response to the comments of nyborg and rooney. J. Acoust. Soc. Am. 1984, 3, $1003-1004$. [CrossRef]

35. Bao, G.; Gao, H.; Cheng, T.H.; Xiao, C.F.; Li, H. Investigation of ultrasonic friction reduction and its development in pneumatic system. Chin. Hydraul. Pneum. 2014, 3, 3171-3190.

36. Qu, J.J.; Zhou, N.N.; Wang, Y.L. Experimental study of air squeeze effect on high-frequency friction contact. Tribol. Int. 2010, 43, 2190-2195. [CrossRef]

(C) 2016 by the authors; licensee MDPI, Basel, Switzerland. This article is an open access article distributed under the terms and conditions of the Creative Commons Attribution (CC-BY) license (http://creativecommons.org/licenses/by/4.0/). 